Article

\title{
Evaluation of the Effects of a Machine Failure on the Robustness of a Job Shop System-Proactive Approaches
}

\section{Iwona Paprocka}

Faculty of Mechanical Engineering, Silesian University of Technology, Konarskiego 18A str., 44-100 Gliwice, Poland; iwona.paprocka@polsl.pl; Tel.: +48-32-327-16-57

Received: 25 November 2018; Accepted: 17 December 2018; Published: 22 December 2018

\begin{abstract}
Researchers are searching for opportunities to organize production systems that save energy and natural resources. Preventive maintenance (PM) is essential for the efficient use of machines and energy saving. Any rework due to a machine failure consumes additional energy, human resources, equipment, spare parts and raw materials. Two criteria-quality robustness $(\mathrm{QR})$ and solution robustness (SR) - are used in order to compute the operational efficiency of the production system in the event of disruption. Any cost criterion can be added to the $\mathrm{QR}$ in order to measure losses due to a machine failure. The SR criterion measures a number of changes necessary to adopt the production schedule after the machine failure. Two proactive approaches are compared to compute the operational efficiency. In the predictive-reactive approach, the PM time is predicted and a stable schedule is built. In the proactive-reactive approach, a schedule is achieved for the best sequence of idle times between jobs. The influence of disturbance on both schedules using robustness measures is examined. This paper presents the results of computer simulations for the above approaches. The approaches are compared in order to select a better method of production organization that reduces costs and waste due to machine failure.
\end{abstract}

Keywords: sustainable manufacturing; robustness; preventive maintenance; proactive scheduling; predictive-reactive scheduling; immune algorithm; job shop

\section{Introduction}

Real-life situations relate to a new way of doing business and creating value by "sustainable manufacturing". Researchers are searching for production systems that minimize negative environmental impacts, conserve energy and natural resources, are safe for employees, communities and consumers, and are economically sound.

Energy saving is a key factor resulting from the need to reduce costs, use limited resources efficiently and be environmentally friendly. Preventive maintenance (PM) is essential for the efficient use of machines and energy saving. Any rework due to a machine failure consumes additional energy, human resources, equipment, spare parts and raw materials. Additional energy is consumed by adapting of a shop floor in accordance with the schedule changes. Additional set-up operations are carried out for machines. Additional organizational changes of human resources and raw materials requirements are also necessary. In other words, the fewer changes in the adopted schedule, the greater the energy savings. A stable and reliable schedule can be obtained using predictive methods for determining PM time. Building a schedule for production and maintenance jobs helps in the creation of manufacturing processes that minimize negative environmental impacts, conserve energy and natural resources, and are economically sound. The number of changes made to the schedules after the disturbance (the bottleneck failure) is measured using the criteria of quality robustness and solution 
robustness. Quality robustness measures the degradation of the performance of the schedule due to the disturbance. Solution robustness measures the sum of absolute deviations of operation start times in the reactive and basic schedules. The impact of machine failures must be minimized.

Preventive maintenance is carried out in order to keep the production system at the high level of operation due to restricted resources. The main idea of PM is to prevent failures before they occur. Some papers search for a method that minimizes the probability of failure. Other papers search for a method that minimizes the impact of failure on a schedule. The first group of methods belongs to predictive scheduling. The second group of methods belongs to proactive and reactive scheduling. Both groups are important in real-life situations. The following literature review is carried out taking into account the above research groups.

\subsection{Literature Review}

First, the pros and cons of predictive, proactive and reactive scheduling methods are determined. The main idea of predictive scheduling is to introduce maintenance into a schedule in order to increase the probability of running the schedule without disturbances. In predictive scheduling, researchers assume that PM performed at regular intervals is enough for machines to be available and reliable [1]. Some researchers assume that the time to implement preventive maintenance is flexible. Certain flexibility is obtained by giving each maintenance task a time window in which the actual start time of the maintenance can move [1,2]. However, more reliable methods are based on inserting a time buffer for maintenance prior to a job with a disturbance prediction [3].

In proactive scheduling, the impact of an unexpected machine failure over the performance of the production system is investigated. A common feature of the proactive scheduling methods is that researchers advocate schedule robustness to deal with uncertainty. A schedule that best deals with the disturbance is accepted for action. There are two types of schedule assessment measures: robustness of a solution and robustness of the solution quality. Solution robustness measures the insensitivity of the operation start times to variations in the input data. Quality robustness measures the insensitivity of objective functions due to disruptions [4]. Proactive scheduling methods differ in their strategy of reducing the impact of uncertainty. Some researchers use prediction methods in order to predict time of PM and built predictive schedules. Next, the influence of the disturbance on the predictive schedule using the robustness measures is examined. This approach is called predictive-reactive (proactive with prediction) [5-7]. Some researchers simply investigate the impact of disruption on a proactive schedule using robustness criteria. But the proactive schedule is achieved for the best sequence of idle times between jobs or batches taking advantage of the simulation process. This approach is called proactive-reactive (proactive without prediction) [8-10]. Predictive-reactive and proactive-reactive approaches are implemented at the decision stage. It is necessary to compare predictive-reactive and proactive-reactive approaches and this is the subject of this paper.

In reactive (dynamic) scheduling, the impact of frequency and method of rescheduling over the performance of a production system is investigated. Researchers investigate when and how to respond to real-time events [11]. The efficiency of rescheduling techniques is assessed using robustness criteria. Reactive scheduling is carried out at runtime to adjust the schedule to the real-time situation.

The author of this paper intends to compare proactive-reactive approaches with predictive-reactive approaches. Thus, the literature review is continued considering these two groups.

First, proactive-reactive approaches are considered. Lei [12] considered PM as an availability constraint but there is no explanation of how the PM time is determined. Each maintenance operation has a fixed predefined time interval. The beginning times of operations are fuzzy. The author examined the impact of PM time on the completion times of jobs. He proved that "most of the possible actual completion times lie in the cut of fuzzy completion time for each job." [12]. Bali and Labdelaoui [13] searched for a maintenance schedule that guarantees a high level of system reliability and reduces both maintenance resources and power demand. The time horizon of the schedule is divided into periods in which maintenance operations are performed. The authors examine the impact of starting times of 
maintenance, for each unit of the power system, on constraints of the problem. The first constraint imposes a continuous and limited maintenance window for each unit of the power system. The second constraint imposes a limited total number of maintenance crew available for each scheduling period. The third constraint imposes a limited capacity of the running units at each period of the maintenance schedule. The capacity should not be less than the predicted load demand at given period.

Considering only the relationship between production and maintenance as a conflict in management decisions may result in unsatisfied demand or machine breakdowns. A common objective is to maximize system productivity and efficiency. Moreover, the time interval of PM activities and the number of PMs are usually pre-known and fixed in advance. In the proactive-reactive approaches, reliability analyses need to be applied to handle the maintenance aspect. The mentioned deficiencies in the proactive-reactive approaches are the advantages in the predictive-reactive approaches.

Next, predictive-reactive approaches are considered. Mokhtari et al. [14] searched for the best allocation of jobs for machines in order to satisfy the predefined makespan value as well as PMs in order to minimize unavailability of the production system. The advantage of the proposed method is the description of a machine condition using the availability function. Unavailability of the machine is zero at the beginning of the scheduling horizon. Availability of the machine is deteriorated with the maximum repair rate predefined for the machine. The machine is restored to the "as good as new" condition after PM. PM time is determined for each machine in order to maximize the availability of the production system. By using the availability component in the objective function, the algorithm assigns PMs in intervals that increase the availability of machines. But the searching process is longer because the neighborhood search algorithm is based on random insertion and swap moves. The disadvantage of this method is the lack of prediction of the time of machine unavailability. Bajestani et al. [15] searched for the best allocation of jobs and PMs for machines in order to minimize the total cost of maintenance and lost production. Two cases are considered: a machine is maintenance-free and the machine needs maintenance. In the first case, the state of the machine and its production rate are known at the beginning of a time period. The transition probability in which the machine changes its state at a given time is predefined. The probability that a machine is in a given state at the start-time of a job depends on both the condition of the machine and the time of maintenance. The disadvantage of the proposed method is that maintenance is performed with a negligible time at the beginning of each time period. The machine is maintained only when it is in a state of failure and as a result, there is no production at the beginning of the next period. The average production rate of the machine depends on its state given at the beginning and at the end of the period. And the average values are described by given time intervals (uniformly distributed) with no historical analysis. In the paper [16] the idea of predictive-reactive scheduling of production tasks is outlined. Predictive scheduling is proposed for a production process with two uncertainties: unknown machine failure times and variability of task processing times. A possible machine failure is introduced as a buffer time (for maintenance) with a fixed duration in the production model. The time of failure is pre-determined for each machine. Failure times are selected based on the assumption of the largest number of tasks processed on machines at that time. The advantage of the method is that the lengths of time buffers are supported by the technical data issued by the maintenance department of a given manufacturing company.

Taking into account machine conditions allows for more efficient use of potential production time. Some predictive-reactive approaches use probability theory to describe machine conditions. Other predictive-reactive approaches assume only a fixed period of unavailability of machines for technical service. However, accepting the assumption that machine conditions are observable at the beginning of each period is not sufficient. Recently, the problems of production scheduling with disturbances have become more and more popular in the scientific community. The most popular machine maintenance strategies are based on the periodic inspection of a machine and age dependent inspection. Researchers should use predictive methods to analyse historical failure-free times. Attributes to describe a machine age and the influence of maintenance should be drawn from analysis of historical data $[7,17]$. 
The advantages of the predictive-reactive method used in this paper are four-fold:

- The predictive-reactive approach uses historical data analysis for PM time prediction;

- Preventive maintenance of the bottleneck is planned in order to reduce unexpected failure;

- Flexible operations are allocated to the bottleneck during a higher probability of failure in order to increase the robustness of the schedule;

- The computer simulation is used to determine the start time of operations on machines in a balanced schedule for robustness criteria.

\subsection{Goals and Approaches}

It is necessary to examine the following research point-which construction of proactive algorithms achieves better solutions: proactive-reactive (proactive without prediction) or predictive-reactive (proactive with prediction). The first construction consists in repeating the closed loop: the set of task sequences is obtained in the coding procedure, trained in the affinity maturation procedure and evaluated after the decoding procedure. The quality of a schedule depends on the impact of the disruption on criteria: the makespan $C_{\max }$, total tardiness $T$, total flow-time $F$, total idle time of machines $I$, and stability criterion SR. The second construction consists in repeating the closed loop: a population of the task sequence is generated, the sequence is rebuilt using the minimal impact of disrupted operation on the schedule MIDOS rule in order to make the schedule more robust. In the MIDOS rule, PM is assigned to the bottleneck at the time of predicted failure. Estimation methods of the mean time to failure and other reliability characteristics are presented in $[7,17]$. Next, the sequences of production and maintenance tasks are trained and evaluated taking into account the effects of the machine failure on criteria: $C_{\max }, T, F, I$ and SR. Taking into account the reliability information of a critical machine some predictions are added in the second construction. Both approaches are compared using the multi-objective immune algorithm described in the next section.

The original contribution to the existing state of research is the comparison analysis of proactive methods using not only the advantage of computer simulation but also prediction. Bearing in mind the review of the literature, the research points are defined:

1. Approaches of dealing with the machine failure and obtaining the best proactive schedules, when the objective is to minimise criteria: $C_{\max }, T, F, I$.

2. Methods dealing with anticipated or unanticipated disruptions, that maximize robustness criteria: SR and QR.

The paper is organized as follows: two constructions of proactive algorithms-proactive-reactive (proactive without prediction) and predictive-reactive (proactive with prediction)-are presented in the next section. The criteria for the assessment of predictive and reactive schedules are also described in Section 2. The mathematical model of the production system with disruptions (a machine failure) is presented in Section 3. The job shop scheduling problem, together with interruptions for experimental study, is presented in Section 4. Section 5 contains necessary analyses and experimental test results related to the research on the application of proactive algorithms in the job shop problem. The paper concludes with a brief summary of the results (Section 6).

\section{Two Proactive Approaches Using the HMOIA}

Originally, the multi-objective immune algorithm (MOIA) was applicable to solve deterministic (basic) scheduling problems [18-20].

Next, modification of the MOIA was applied to solve proactive-reactive scheduling problems [21]. The modification is called the hybrid multi-objective immune algorithm II (HMOIA II). The HMOIA II searches the solution space for the most stable and robust solutions. The algorithm repeats the procedures: coding solutions, training solutions, decoding solutions, evaluating solutions and eliminating similar solutions (Figure 1). The algorithm effectively scans the solution space to achieve 
the most robust schedule (Fig.1 in [21]). Solutions are evaluated using two criteria: solution robustness (SR) and quality robustness (QR). QR measures how much the quality of the basic schedule has deteriorated after the disruption. SR measures the sum of absolute deviations of operation start times in the reactive and nominal (basic) schedule. The fitness function adds the weighted values of $Q R$ and SR. The criteria are equivalent.

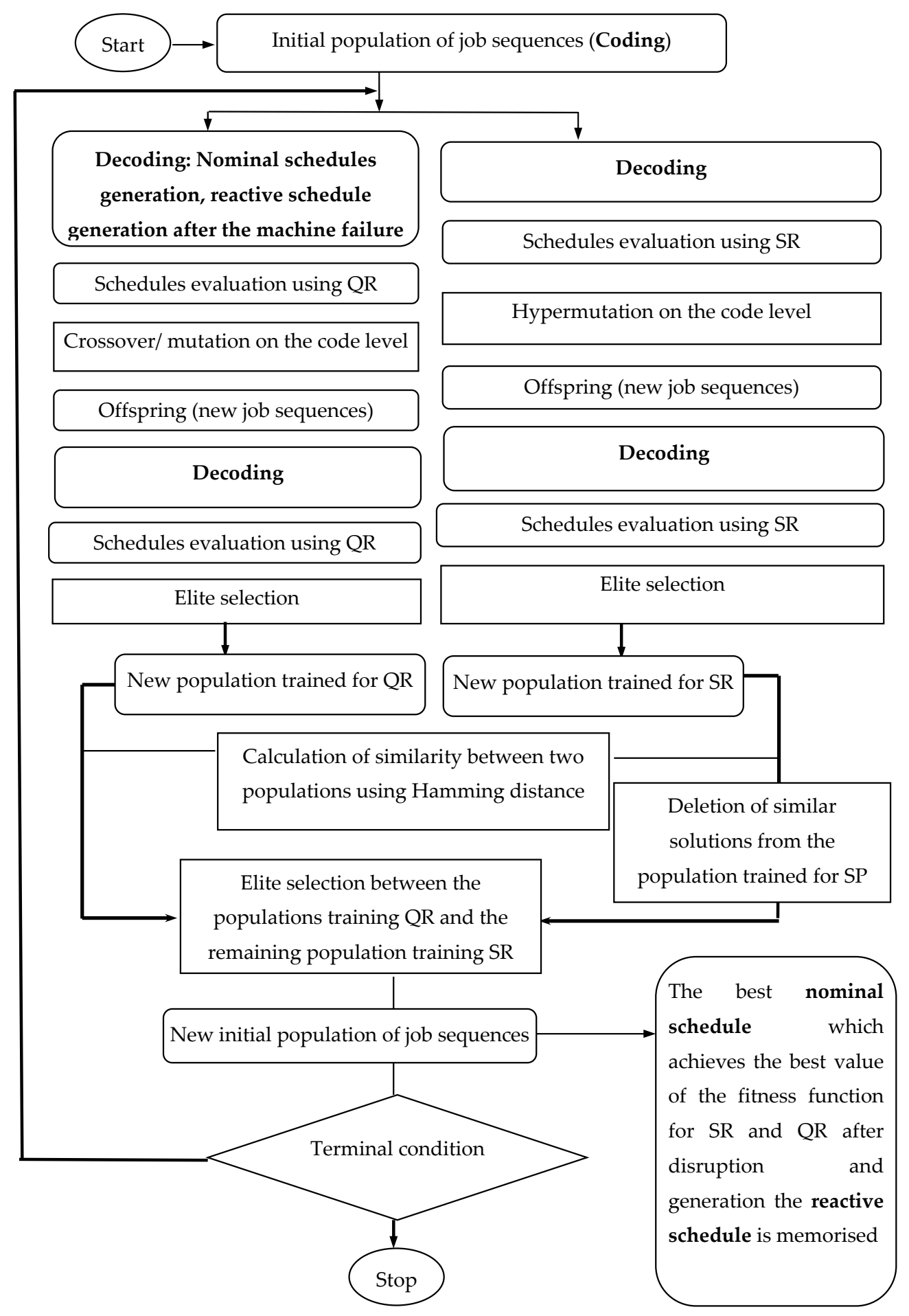

Figure 1. Architecture of the hybrid multi-objective immune algorithm (HMOIA) II in the searching process for the best nominal schedule that will best deal with disruption. 
The elite selection is used to select a better solution from a pair: parent and offspring. Solutions with lower values of the fitness function create a new initial population in the next iteration. In each iteration, the best solution is copied to the immune memory. It means that the job sequence will best deal with disruption (Figure 1). In HMOIA II, the training process is terminated with a given number of iterations.

“The training process of antibody population for QR starts with antibodies selection to create a mating pool. Parents are randomly matched in couples." [21] (Figure 1). Mutation and crossover procedures are used to train the population of solutions for QR [21]. Hypermutation procedure is used to train the population of solutions for SR [21].

In order to maintain a high diversity of solutions trained in subpopulations for QR and SR, the Hamming distance is calculated for each solution. Affthres is a threshold value that determines the similarity of two solutions. "An antibody is deleted from the population trained for the SR if it is stimulated by a number of antibodies more than stimthres." [21]. The removed antibody is replaced by an antibody with the same index from the population trained for SR.

After the terminal condition is met, the best solution is selected from the immune memory.

In [21], authors proposed the construction of the algorithm to achieve the best compromise solution for SR and QR. In this paper, the decoding process is differentiated in two approaches: proactive-reactive (proactive without prediction) or predictive-reactive (proactive with prediction) (Figures 2 and 3).

Decoding 1: Generating a nominal schedule

Indication of interrupted operations after the bottleneck failure

Delete the interrupted operations and subsequent operations of the interrupted task

Plan a repair task in the bottleneck during a real failure

For each disrupted task, assign disrupted operations to machines available first

Figure 2. The decoding process in the proactive scheduling approach without prediction: generating a nominal schedule (HMOIA II).

In the first approach (HMOIA II), a job sequence is decoded by generating the nominal schedule (with deterministic input data), then, the reactive schedule is generated after the bottleneck failure (Figure 2). In the second approach (HMOIA III), a job sequence is decoded by generating the basic (nominal) schedule. The basic schedule is then rebuilt using the MIDOS rule in order to generate a predictive schedule [22]. The MIDOS rule assigns a preventive maintenance task at the predicted time of the bottleneck failure. Then the most flexible task operations are assigned during the high probability period of failure. The MIDOS rule makes the schedule more robust and flexible in the event of the bottleneck failure. The reactive schedule is generated after the bottleneck failure (Figure 3).

The proactive scheduling approaches differ in their strategy of reducing the impact of uncertainty. The first approach (HMOIA II) simply investigates the impact of disruption on a nominal schedule using robustness criteria. But the proactive schedule is achieved for the best sequence of jobs taking advantage of the simulation process. The second approach (HMOIA III) uses prediction methods $[7,17]$ in order to predict time of PM and built a predictive schedule using the MIDOS rule. Then, the influence of the disturbance on the predictive schedule using the robustness measures is examined.

Computer simulations are run for the two proactive approaches for the job shop scheduling problem and reliability characteristics presented in Section 4 . First the mathematical model of the production system with disruptions (a machine failure) is presented. 
Decoding 2: Generating a basic schedule

Indication of tasks that may be interrupted in the event of the bottleneck failure

Delete the tasks that are expected to be disrupted

Assigning preventive maintenance in the bottleneck in the foreseeable time of failure

For each deleted task:

Compute the MIDOS coefficient for each operation that can be performed in the bottleneck as a parallel machine

The assignment of the operation that is the most flexible (the highest value of the MIDOS) in the bottleneck during the increased probability of failure if the machine is idle

Assigning operations preceding the most flexible operation to machines available first in the backward scheduling procedure

Assigning operations succeeding the most flexible operation to machines available first in the forward scheduling procedure

Indication of interrupted operations after the bottleneck failure

Delete the interrupted operations and subsequent operations of the interrupted task

Plan a repair task in the bottleneck during a real failure

For each disrupted task, assign disrupted operations to machines available first

Indication of interrupted operations after the bottleneck failure

Delete the interrupted operations and subsequent operations of the interrupted task

Block the bottleneck in time of failure

Figure 3. The decoding process in the proactive scheduling approach with prediction (HMOIA III).

\section{The Mathematical Model of the Production System with Disruptions}

A job shop scheduling problem with disturbance is described by $J \operatorname{tasks}(j=1,2, \ldots, J)$ that are to be scheduled on $W$ machines $(w=1,2, \ldots, W)$. Each production task is represented by a number of non-pre-emptive operations $V_{j}$ which is equal to the number of machines $\left(v_{j}=1,2, \ldots, V_{j}\right)$. The execution of operation $v_{j}$ of task $j$ requires a machine according to a technological route. Operation $v_{j}$ occupies predefined machine time units. After the machine failure, operation $v_{j}$ requires one machine selected from a set of parallel machines. Processing time $a_{w, v_{j}}$ of operation $v_{j}$ is non-resumable after a disruption (the bottleneck failure). It means that operation $v_{j}$ must be reprocessed fully after repair of the bottleneck.

The maintenance task must be performed on the most occupied machine in every planning horizon. The production system with disturbances (bottleneck failures) is monitored in order to collect information on a number of disruptions, disruption-free times and repair times. It is assumed that successive disruption-free times are assumed to have Weibull distributions and are followed by exponentially distributed times of machine service (repair). It was observed that parameters of these distributions change with time. Based on the collected information in a number of planning horizons 
of the same duration in the past predictions of the reliability characteristics are estimated. Such a production system, firstly, is observed on $m$ successive planning horizons:

$$
[0, T),[T, 2 T), \ldots,[(m-1) T, m T)
$$

of the same duration, for which information about numbers of disturbances or disturbance-free times is collected. The prediction of job shop system operation is being built for the next planning horizon $[m T,(m+1) T)$. Disturbance-free times $X_{i, 1}, \ldots, X_{i, N_{i}}$ in the $i$ th planning horizon $[(i-1) T, i T), i=$ $1, \ldots, m+1$ have a Weibull distribution with probability density function $f_{i}(\cdot)$ of the form:

$$
f_{i}(t)=\left\{\begin{array}{l}
\lambda_{i} p_{i} t^{p_{i}-1} \exp \left(-\lambda_{i} t^{p_{i}}\right), t>0 \\
0, t \leq 0
\end{array}\right.
$$

where $p_{i}>0, \lambda_{i}>0$, thus parameters of the distribution depend on the number of period and are the same in each period separately. Here $N_{i}$ denotes a random number of failures detected in $[(i-1) T, i T)$.

At the end of reliable work period $X_{i, k}$, as the failure occurs, a repair time $Y_{i, k}$ begins immediately and so on. Repair times $Y_{i, 1}, \ldots, Y_{i, N_{i}}$ for $i=1, \ldots, m+1$ are supposed to be exponentially distributed with probability distribution functions $\operatorname{PDFs} g_{i}(\cdot)$ of the form:

$$
g_{i}(t)=\left\{\begin{array}{l}
\alpha_{i} \exp \left(-\alpha_{i} t\right), t>0 \\
0, t \leq 0
\end{array}\right.
$$

Assuming that numbers and durations of successive disturbance-free periods have been measured and are known for each planning horizon, parameters of distributions $f_{i}(\cdot)$ are estimated using the maximum likelihood approach or empirical moments approach.

\subsection{Maximum Likelihood Approach}

Assuming that in each of planning horizon $[0, T),[T, 2 T), \ldots,[(m-1) T, m T)$ disturbance-free periods $X_{i, k}, k=1, \ldots, N_{i}$ for any $i=1, \ldots, m$ are given, where $n_{i}$ is the observed value of $N_{i}$, thus the observations are described by:

$$
\left(x_{1,1}, x_{1,2}, \ldots, x_{1, n_{1}}\right), \ldots,\left(x_{m, 1}, x_{m, 2}, \ldots, x_{m, n_{m}}\right)
$$

Consider firstly the planning horizon $[0, T) . x_{1,1}, x_{1,2}, \ldots, x_{1, n_{1}}$ are independent and identically distributed collected variables. Unknown parameter $p_{1}$ of Weibull distribution is estimated using the following equation [23]:

$$
n \sum_{k=1}^{n_{1}} x_{1, k}^{p_{1}} \ln x_{1, k}-\left(\frac{n_{1}}{p_{1}}+\sum_{k=1}^{n_{1}} \ln x_{1, k}\right) \sum_{k=1}^{n_{1}} x_{1, k}^{p_{1}}=0 .
$$

Obtaining estimated value of $\hat{p_{1}}$ unknown parameter $\lambda_{1}$ of Weibull distribution is estimated just from the following equation:

$$
\hat{\lambda_{1}}=\frac{n_{1}}{\sum_{k=1}^{n_{1}} x_{1 k}^{\hat{\hat{p}_{1}}}}
$$

After achieving estimators $\hat{p_{1}}, \ldots, \hat{p_{m}}$ and $\hat{\lambda_{1}}, \ldots, \hat{\lambda_{m}}$ one can extrapolate values $\hat{p}_{m+1}$ and $\hat{\lambda}_{m+1}$ for the next planning horizon $[m T,(m+1) T)$ for which we have no observation, using the regression method. 


\subsection{Empirical Moments Approach}

Assume that there are disturbance-free periods $x_{1,1}, x_{1,2}, \ldots, x_{1, n_{1}}$ for planning horizon $[0, T)$ as in the previous section. Analyzing the behavior of the condition [23]:

$$
T_{1}\left(\frac{1}{p_{1}}\right)=\sqrt{\frac{\pi}{p_{1}}} \cdot\left(\frac{1}{4}\right)^{\frac{1}{p_{1}}}
$$

for fixed value $T_{0}=\frac{m_{1}^{2}\left(x_{1}\right)}{m_{2}\left(x_{1}\right)}$, one can find an approximate value $\hat{p_{1}}$ of $p_{1}$. Here $m_{1}\left(x_{1}\right)$ denotes disturbance-free mean and $m_{2}\left(x_{1}\right)$ is the second moment as follows:

$$
m_{1}\left(x_{1}\right)=\bar{x}_{1}=\frac{1}{n_{1}} \sum_{k=1}^{n_{1}} x_{1, k}, m_{2}\left(x_{1}\right)=\frac{1}{n_{1}} \sum_{k=1}^{n_{1}} x_{1, k}^{2}
$$

Having $\hat{p_{1}}$ one can obtain an approximate value $\hat{\lambda_{1}}$ of $\lambda_{1}$ just from the equation:

$$
\hat{\lambda_{1}}=\left[m_{1}\left(x_{1}\right) / \Gamma\left(\frac{1}{\hat{p_{1}}}+1\right)\right]^{-\hat{p_{1}}}
$$

The exact value of $\lambda_{1}$ is impossible to obtain just from the above equation. One method of approximation uses the Gamma function:

$$
\Gamma(x+1) \approx x^{x+\frac{1}{2}} e^{-x} e^{\frac{1}{2} \ln 2 \pi}, x \rightarrow \infty
$$

After obtaining estimators $\hat{p_{1}}, \ldots, \hat{p_{m}}$ and $\hat{\lambda_{1}}, \ldots, \hat{\lambda_{m}}$ one can extrapolate values $\hat{p}_{m+1}$ and $\hat{\lambda}_{m+1}$ for the next planning horizon $[m T,(m+1) T)$ for which we have no observation, using the regression method.

\subsection{Reliability Characeristics}

Let us consider planning horizons $[(m-1) T, m T)$ for which estimators $\hat{p}_{m+1}$ and $\hat{\lambda}_{m+1}$ of Weibull distribution were obtained (using one of two methods). Below the formulae for the reliability characteristics used in the MIDOS rule are given:

1. The maintenance task is assigned to the bottleneck at the time determined by the mean time between failures (MTBF)

$$
M T B F=E\left\{X_{m+1,1}+Y_{m+1,1}\right\}=\left(\frac{1}{\hat{\lambda}_{m+1}}\right)^{\frac{1}{\hat{p}_{m+1}}} \Gamma\left(\frac{1}{\hat{p}_{m+1}}+1\right)+\left(\frac{1}{\alpha_{m+1}}\right),
$$

where: $\alpha_{m+1}>0$ is predefined.

2. The risk of inconsistent prediction of machine failure-free time is minimized by assigning the most flexible operations to the period limited by points $b$ and $a$, where: $a$ is estimated on the assumption [7] that the probability of the failure-free time of the bottleneck described by the Weibull distribution with parameters $\hat{p}_{m+1}$ and $\hat{\lambda}_{m+1}$ is higher than $a$ equalling $40 \%$, $P\left\{X_{m+1,1}>a\right\}=0.4$. Thus, $P\left\{X_{m+1,1}<a\right\}=0.6$ and $1-e\left(-\lambda \cdot a^{p}\right)=0.6$, and we have $e\left(-\lambda \cdot a^{p}\right)=0.4$ and taking the property $\ln e(r)=r$ we achieve $a^{p}=-\ln (0.4) / \lambda$ and finally:

$$
a=\sqrt[p]{-\ln (0.4) / \lambda}
$$

$b$ is estimated on the assumption that the probability of the failure-free time of the bottleneck described by Weibull distribution with parameters $\hat{p}_{m+1}$ and $\hat{\lambda}_{m+1}$ is less than $b$ equalling $70 \%$, 
$P\left\{X_{m+1,1}<b\right\}=0.7$ thus $1-e\left(-\lambda \cdot b^{p}\right)=0.7$ and we have $e\left(-\lambda \cdot b^{p}\right)=0.3$ and taking the property $\ln e(r)=r$ we achieve $b^{p}=-\ln (0.3) / \lambda$ and finally

$$
b=\sqrt[p]{-\ln (0.3) / \lambda}
$$

Having the above reliability characteristics, a flexible schedule is generated using the MIDOS rule. The achieved schedule is evaluated before and after the disturbance.

\subsection{Evaluation Criteria}

The job shop scheduling problem with disturbance is formulated to schedule a set of production tasks to a set of machines subject to the following constraints:

- the operation sequence of a production task is predefined;

- operations of production tasks are pre-assigned to machines according to technological routes;

- the disturbed operation can be rescheduled to one machine from a set of parallel machines;

- preventive maintenance of the bottleneck has to be completed at the time of the MTBF.

The objective is to minimise the following criteria of predictive schedule $\mathrm{x}$ :

1. Makespan of production tasks:

$$
F_{1}=\min C_{\max \left[t_{z, V_{j}}\right] \max }
$$

where: $t_{z, v_{j}}$ is completion time of operation $v_{j}$ of task $j, v_{j}=1, \ldots, V_{j}, j=1, \ldots, J$;

2. Flow time of production tasks:

$$
F_{2}=\min F=\sum_{j=1}^{J}\left(t_{z, V_{j}}-t_{r, 1_{j}}\right)
$$

where: $t_{r, v_{j}}$ is start time of operation $v_{j}$ of task $j$;

3. Total tardiness of production tasks:

$$
F_{3}=\min T=\sum_{j=1}^{J}\left[0, D_{j}\right]
$$

where: $\left\{\begin{array}{c}0, \text { if } d_{j}-t_{z, V_{j}} \leq 0 \\ D_{i}, \text { if } d_{j}-t_{z, V_{j}}>0\end{array}\right.$ and $d_{j}$ is deadline for completing task $j ; D_{j}$ is delay in completing task $j$.

4. Idle time of machines:

$$
F_{4}=\min I=\sum_{w=1}^{W} I_{w}
$$

where $I_{w}$ is idle time of machine $w, w=1, \ldots, W$.

5. The weighted sum of values of the four objective functions are combined into one objective function:

$$
F F(x)=\omega_{1} \cdot F_{1}(x)+\omega_{2} \cdot F_{2}(x)+\omega_{3} \cdot F_{3}(x)+\omega_{4} \cdot F_{4}(x)
$$

Subject to:

$$
\omega_{1}, \omega_{2}, \omega_{3}, \omega_{4} \in[0,1] \text {, and } \mathfrak{\omega}_{1}+\omega_{2}+\omega_{3}+\omega_{4}=1
$$

The objective is to minimise the following criteria of reactive schedule $x^{*}$ : 
1. The weighted sum of values of the four objective functions:

$$
F F(x *)=\omega_{1} \cdot F_{1}(x *)+\omega_{2} \cdot F_{2}(x *)+\omega_{3} \cdot F_{3}(x *)+\omega_{4} \cdot F_{4}(x *)
$$

2. Quality robustness:

$$
Q R(x *)=|F F(x)-F F(x *)|
$$

3. Solution robustness:

$$
S R(x *)=\sum_{j=1}^{J} \sum_{v_{j}=1}^{V_{j}}\left|s t_{j, v_{j}}(x)-s t_{j, v_{j}}(x *)\right|
$$

where $s t_{j, v_{j}}(x)$ is start time of operation $v_{j}$ of task $j$ in predictive schedule $\mathrm{x}$; and $s t_{j, v_{j}}(x *)$ is start time of operation $v_{j}$ of task $j$ in reactive schedule $x^{*}$.

4. The weighted sum of values of the above robustness criteria:

$$
\operatorname{FFr}(x *)=\omega_{1} \cdot Q R_{1}(x *)+\omega_{2} \cdot S R_{2}(x *)
$$

Subject to:

$$
\omega_{1}, \omega_{2} \in[0,1], \text { and } \omega_{1}+\omega_{2}=1
$$

\section{A Job Shop Scheduling Problem}

A numerical example of a job shop (JS) system with disturbances is presented in this section. A total of 15 production tasks should be executed on 10 machines $(15 \times 10)$ in the JS scheduling problem. Routes of the production processes, durations of technological operations, deadlines of production tasks and a set of parallel machines are presented in [24]. The goal is to obtain a feasible solution for four criteria: $C_{\max } \rightarrow \min ; F \rightarrow \min ; T \rightarrow \min$ and $I \rightarrow \min$. The weights of the criteria are as follows: $C_{\max }$ and $T$ equal 0.3; $F$ and $I$ equal 0.2. "The increased probability of the bottleneck failure occurs in time horizon: $[a, b+M T T R]$ where: $a=60$ and $b=72$ and the mean time of repair, MTTR $=6$. The mean time to failure, MTTF) equals 66" [24].

The question is: Which structure of proactive algorithms allows one to achieve better solutions: proactive-reactive (proactive without prediction) or predictive-reactive (proactive with prediction)? In the two proactive approaches, the population of task sequences is trained and then evaluated taking into account the effects of the disturbance. The effect of the disturbance is assessed using the SR and QR.

In the proactive-reactive approach, the criterion used for stability (SR) is the sum of absolute deviations between the start times of operations of planned jobs in the nominal schedule and those performed after the disruption (in the reactive schedule). In the predictive-reactive approach, the criterion used for stability (SR) is the sum of absolute deviations between start times of operations of planned jobs in the predictive schedule and those performed after the disruption (in the reactive schedule).

In the proactive-reactive approach, the criterion of quality robustness $(\mathrm{QR})$ is the difference between the fitness function value (FFy) obtained for the nominal schedule $y$ and the fitness function value $\left(\mathrm{FFy}^{*}\right)$ achieved for reactive schedule $\mathrm{y}^{*}$. In the predictive-reactive approach, the criterion of quality robustness $(\mathrm{QR})$ is the difference between the fitness function value (FFx) obtained for the predictive schedule $x$ and the fitness function value $\left(\mathrm{FFx}^{*}\right)$ achieved for reactive schedule $x^{*}$. In both proactive approaches, it is possible to use any combination of criteria $C_{\max }, F, I$ and $T$ for assessing the schedules before and after the disruption [13]. The fitness function (FFy) adds the weighted values of the criteria: $C_{\max }, F, I$ and $T$ achieved for the nominal schedule $y$ in the proactive -reactive approach. The fitness function (FFx) adds the weighted values of criteria $C_{\max }, F, I$ and $T$ achieved for the predictive schedule $x$ in the predictive-reactive approach. The fitness function achieved for 
reactive schedule $\left(\mathrm{FFy}^{*}\right.$ or $\left.\mathrm{FFx}^{*}\right)$ adds the weighted values of criteria $C_{\max }, F, I$ and $T$ achieved for the reactive schedule in both proactive approaches. Weights of criteria $C_{\max }, F, I$ and $T$ are predefined depending on decision-maker preference.

Also, the weighted sum of SR and QR is computed for the predictive-reactive approach (FFrx*) and for the proactive-reactive approach (FFry ${ }^{*}$ ). The weights of the two criteria are equal.

In the next section, the results of computer simulations of the application of the two proactive approaches are presented. The influence of the quality of basic schedules over the quality of reactive schedules is investigated in the proactive-reactive approach. The influence of the quality of predictive schedules over the quality of reactive schedules is investigated in the predictive-reactive approach. The objective is to find an approach that is able to generate a stable and robust schedule in the event of the bottleneck failure.

\section{Results of Computer Simulations}

In the HMOIA II and III, input parameters have the same values in order to allow comparison. The input parameters are presented in [21]. Both algorithms were coded in Borland C++.

\subsection{Predictive-Reactive (Proactive with Prediction) Approach (HMOIA III)}

Searching for the best predictive schedule for the JS problem using the HMOIA III, six computer simulations were carried out for affthres $=8$ and for affthres $=80$. The decision maker determines the number of computer simulations performed. Basic schedules are subject to modification using the MIDOS rule in order to build a predictive schedule. The predictive schedule undergoes a disruption. The schedule is then rebuilt in order to evaluate the effect of the disturbance on the quality of the predictive schedule. The best predictive schedule is obtained based on the minimal value of fitness function FFrx* of reactive schedule The fitness function has two sub-functions: SR and QR.

Let us consider the predictive schedule achieved in the first simulation done for affthres $=8$. The best predictive schedule was obtained for the priority rule of $\{414907836121011325$ 11\}. The quality of the reactive schedule is $\mathrm{FFr}_{1^{*}}=3,3$ with the components of $\mathrm{QR}=0.6$ and $\mathrm{SR}=6$. The quality of this solution is also evaluated using $C_{\max }, F, I$ and $T$. The quality of the predictive schedule is $C_{\max }=125, F=503, I=712$ and $T=127$, and $C_{\max }=125, F=509, I=709$ and $T=127$ after disruption. The rest of the solutions are presented in Table 1.

Table 1. Predictive and reactive schedules achieved by the HMOIA III and affthres $=8$.

\begin{tabular}{|c|c|c|c|c|c|c|c|c|c|c|c|c|c|c|}
\hline \multirow{3}{*}{ No } & \multicolumn{14}{|c|}{ Job Shop Scheduling Problem $(15 \times 10)$} \\
\hline & \multirow{2}{*}{$\begin{array}{c}\text { The Priority Rule } \\
\text { of the Basic } \\
\text { Schedule }\end{array}$} & \multicolumn{5}{|c|}{$\begin{array}{c}\text { The Quality of Predictive } \\
\text { Schedule X }\end{array}$} & \multicolumn{8}{|c|}{ The Quality of Reactive Schedule $x^{*}$} \\
\hline & & $C_{\max }$ & $F$ & $I$ & $T$ & FFx & $C_{\max }$ & $F$ & $I$ & $T$ & $\mathrm{FFx}^{*}$ & QR & SR & FFrx $^{*}$ \\
\hline 1 & $\begin{array}{c}41490783612 \\
101132511\end{array}$ & 125 & 503 & 712 & 127 & 318.6 & 125 & 509 & 709 & 127 & 319.2 & 0.6 & 6 & 3.3 \\
\hline 2 & $\begin{array}{c}651011312487 \\
20913114\end{array}$ & 129 & 647 & 752 & 65 & 338 & 129 & 646 & 749 & 64 & 336.9 & 1,1 & 14 & 7.55 \\
\hline 3 & $\begin{array}{c}147691121180 \\
31013524\end{array}$ & 132 & 574 & 782 & 161 & 359.1 & 132 & 575 & 779 & 156 & 357.2 & 1,9 & 12 & 6.95 \\
\hline 4 & $\begin{array}{c}281312391157 \\
14010164\end{array}$ & 127 & 474 & 732 & 84 & 304.5 & 124 & 468 & 699 & 73 & 292.5 & 12 & 40 & 26 \\
\hline 5 & $\begin{array}{c}128101453206 \\
71141139\end{array}$ & 123 & 589 & 692 & 25 & 300.6 & 123 & 589 & 689 & 25 & 300 & 0,6 & 16 & 8.3 \\
\hline \multirow[t]{2}{*}{6} & $\begin{array}{c}951210117132 \\
81410346\end{array}$ & 127 & 627 & 732 & 58 & 327.3 & 127 & 633 & 729 & 64 & 329.7 & 2,4 & 58 & 30.2 \\
\hline & Average & & & & & 324.68 & & & & & 322.58 & 3.1 & 24.3 & 13.71 \\
\hline
\end{tabular}

The same number of simulations were also run for affthres $=80$. In the fifth simulation, the best predictive schedule was obtained for the priority of $\{81075122141611341309\}$. The quality of the reactive schedule is $\mathrm{FFr}_{5}=5.83$ with the components of $\mathrm{QR}=1.2$ and $\mathrm{SR}=10.5$. The quality of this 
solution is also evaluated using $C_{\max }, F, I$ and $T$. The quality of the predictive schedule is $C_{\max }=122$, $F=557, I=682$ and $T=12$, and $C_{\max }=122, F=554, I=679$ and $T=12$ after disruption. The rest of the solutions are presented in Table 2.

Table 2. Predictive and reactive schedules achieved by the HMOIA III and affthres $=80$.

\begin{tabular}{|c|c|c|c|c|c|c|c|c|c|c|c|c|c|c|}
\hline \multirow{3}{*}{ No } & \multicolumn{14}{|c|}{ Job Shop Scheduling Problem $(15 \times 10)$} \\
\hline & \multirow{2}{*}{$\begin{array}{c}\text { The Priority Rule } \\
\text { of the Basic } \\
\text { Schedule }\end{array}$} & \multicolumn{5}{|c|}{$\begin{array}{c}\text { The Quality of Predictive } \\
\text { Schedule X }\end{array}$} & \multicolumn{8}{|c|}{ The Quality of Reactive Schedule $x^{*}$} \\
\hline & & $C_{\max }$ & $F$ & $I$ & $T$ & FFx & $C_{\max }$ & $F$ & $I$ & $T$ & $\mathrm{FFx}^{*}$ & QR & SR & FFri $^{*}$ \\
\hline 1 & $\begin{array}{c}910111326580 \\
12713144\end{array}$ & 130 & 700 & 769 & 68 & 353.2 & 130 & 704 & 759 & 68 & 352 & 1.2 & 18 & 9.6 \\
\hline 2 & $\begin{array}{c}113871251409 \\
13141062\end{array}$ & 131 & 624 & 772 & 97 & 347.6 & 131 & 619 & 769 & 97 & 346 & 1.6 & 30 & 15.8 \\
\hline 3 & $\begin{array}{c}1413365118102 \\
4011279\end{array}$ & 128 & 472 & 742 & 79 & 304.9 & 126 & 467 & 719 & 69 & 295.7 & 9.2 & 28 & 18.6 \\
\hline 4 & $\begin{array}{c}61004231497 \\
118513112\end{array}$ & 130 & 567 & 762 & 89 & 331.5 & 130 & 567 & 759 & 89 & 330.9 & 0.6 & 23 & 11.8 \\
\hline 5 & $\begin{array}{c}810751221416 \\
11341309\end{array}$ & 122 & 557 & 682 & 12 & 288 & 122 & 554 & 679 & 12 & 286.8 & 1.2 & 10.5 & 5.85 \\
\hline 6 & $\begin{array}{c}02358121964 \\
137111410\end{array}$ & 126 & 614 & 722 & 0 & 305 & 126 & 599 & 719 & 0 & 301.4 & 3.6 & 23 & 13.3 \\
\hline & Average & & & & & 321.7 & & & & & 318.8 & 2.9 & 22.08 & 12.49 \\
\hline
\end{tabular}

Let us compare all the simulations carried out by the HMOIA III. The best schedule for dealing with the uncertainty (the best value of $\mathrm{FFrx}^{*}$ ) was achieved in the first simulation for affthres $=8$. The best predictive schedule was obtained by the HMOIA III (Figure 4).

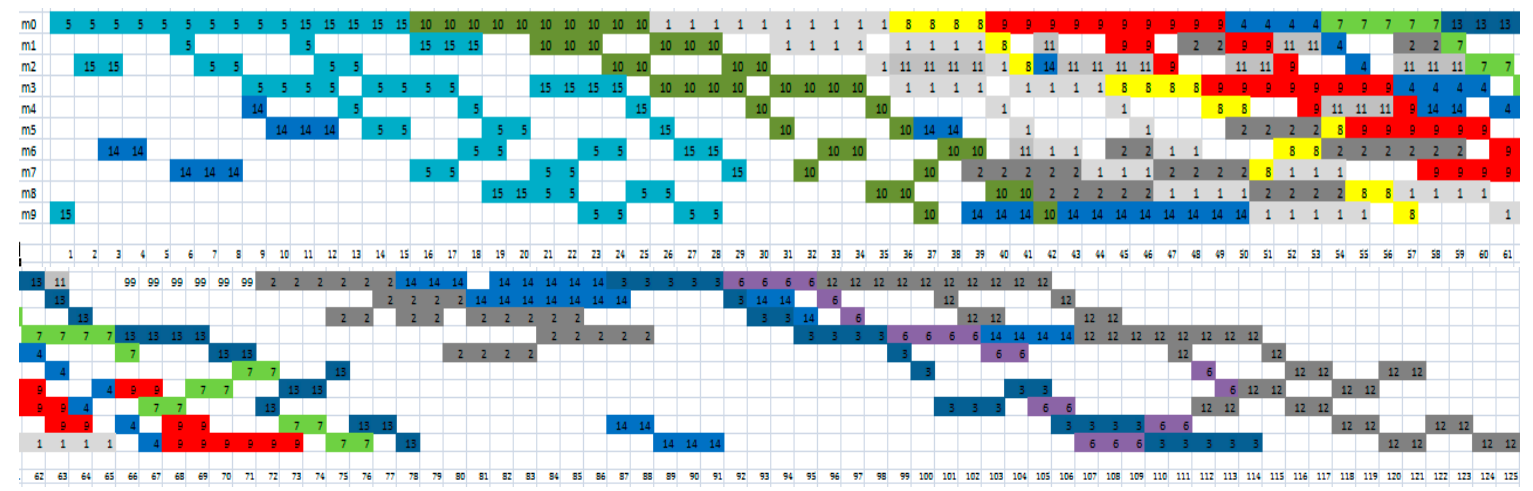

Figure 4. The best predictive schedule obtained by the MOIA III (affthres $=8$ ) and MIDOS where $(i=\{0,2, \ldots, 9\}$ is no. of machine, $(j=\{1,2, \ldots, 15\}$ is no. of job, and 99 is predicted technical inspection at time 66 .

Slightly better predictive schedules were achieved for affthres $=80$ taking into account the average values of FFrx* The same conclusion can be given taking into account the average values of FFx for predictive schedules and $\mathrm{FFx}^{*}$ for reactive schedules.

\subsection{Proactive-Reactive (Proactive without Prediction) Approach (HMOIA II)}

Searching for the best predictive schedule for JS problem using the HMOIA II, six computer simulations were carried out for affthres $=8$ and for affthres $=80$. The basic schedules are subject to disruption. The basic schedules are then rebuilt for the purpose of evaluation the effect of the disturbance on the quality. The best basic schedule $y$ is obtained based on the minimal value of fitness function FFry* of reactive schedule $y^{*}$. The fitness function has two sub-functions: SR and QR.

Consider the best basic schedule achieved for affthres $=8$. The best basic schedule was generated in the fourth simulation. The best basic schedule was obtained for the priority rule of $\left\{\begin{array}{l}3012131084 \\ 0\end{array}\right.$ 
1159261714 . The quality of the reactive solution is $\mathrm{FFr}_{4^{*}}=30.1$ with the sub-functions of $\mathrm{QR}=15.2$ and $S R=45$. The quality of this solution is also measured using $C_{\max }, F, I$ and $T$. The quality of the basic solution is $C_{\max }=129, F=468, I=758$ and $T=157$, and $C_{\max }=127, F=548, I=732$ and $T=139$ after disruption. The rest of the solutions are presented in Table 3.

Table 3. Proactive-reactive schedules achieved by the HMOIA II and affthres $=8$.

\begin{tabular}{|c|c|c|c|c|c|c|c|c|c|c|c|c|c|c|}
\hline \multirow{3}{*}{ No } & \multicolumn{14}{|c|}{ Job Shop Scheduling Problem $(15 \times 10)$} \\
\hline & \multirow{2}{*}{$\begin{array}{l}\text { The Priority Rule of } \\
\text { the Basic Schedule }\end{array}$} & \multicolumn{5}{|c|}{ The Quality of Basic Schedule y } & \multicolumn{8}{|c|}{ The Quality of the Reactive Schedule $y^{*}$} \\
\hline & & $C_{\max }$ & F & $\mathbf{I}$ & $\mathbf{T}$ & FFy & $C_{\max }$ & $\mathbf{F}$ & $\mathbf{I}$ & $\mathbf{T}$ & FFy $^{*}$ & QR & SR & FFry* \\
\hline 1 & $\begin{array}{c}12121410461139 \\
513870\end{array}$ & 131 & 520 & 778 & 22 & 305.5 & 131 & 535 & 772 & 78 & 324.1 & 18.6 & 61 & 39.8 \\
\hline 2 & $\begin{array}{c}1086511312947 \\
0214131\end{array}$ & 133 & 587 & 798 & 100 & 346.9 & 133 & 586 & 792 & 94 & 343.7 & 3.2 & 105 & 54.1 \\
\hline 3 & $\begin{array}{c}13012109154147 \\
211368\end{array}$ & 127 & 539 & 738 & 107 & 325.6 & 127 & 559 & 732 & 107 & 328.4 & 2.8 & 102 & 52.4 \\
\hline 4 & $\begin{array}{c}30121310841159 \\
261714\end{array}$ & 129 & 568 & 758 & 157 & 351 & 127 & 548 & 732 & 139 & 335.8 & 15.2 & 45 & 30.1 \\
\hline 5 & $\begin{array}{c}2501263748910 \\
1311141\end{array}$ & 131 & 577 & 778 & 31 & 319.6 & 131 & 595 & 772 & 31 & 322 & 2.4 & 63 & 32.7 \\
\hline \multirow[t]{2}{*}{6} & $\begin{array}{c}3405912101412 \\
6111387\end{array}$ & 125 & 564 & 718 & 49 & 308.6 & 125 & 581 & 712 & 49 & 310.8 & 2.2 & 120 & 61.1 \\
\hline & Average & & & & & 326.2 & & & & & 327.46 & 7.4 & 82.66 & 45.03 \\
\hline
\end{tabular}

Also, six simulations were generated for affthres $=80$. The best basic schedule was achieved in the first simulation. The best basic schedule was obtained for the priority rule of $\{90117610524128$ 141331 \}. The quality of the reactive solution is $\mathrm{FFr}_{1^{*}}=29.3$ with the sub-functions of $Q R=0.6$ and $\mathrm{SR}=58$. The quality of this solution is also measured using $C_{\max }, F, I$ and $T$. The quality of the basic solution is $C_{\max }=134, F=586, I=808$ and $T=95$, and $C_{\max }=134, F=589, I=802$ and $T=95$ after disruption. The rest of the solutions are presented in Table 4.

Table 4. Proactive-reactive schedules achieved by the HMOIA II and affthres $=80$.

\begin{tabular}{|c|c|c|c|c|c|c|c|c|c|c|c|c|c|c|}
\hline \multirow{3}{*}{ No } & \multicolumn{14}{|c|}{ Job Shop Scheduling Problem $(15 \times 10)$} \\
\hline & \multirow{2}{*}{$\begin{array}{l}\text { The Priority Rule of } \\
\text { the Basic Schedule }\end{array}$} & \multicolumn{5}{|c|}{ The Quality of Basic Schedule y } & \multicolumn{8}{|c|}{ The Quality of Reactive Schedule $y^{*}$} \\
\hline & & $C_{\max }$ & $\mathbf{F}$ & I & $\mathbf{T}$ & FFy & $C_{\max }$ & F & I & $\mathbf{T}$ & FFy $^{*}$ & QR & SR & FFry* \\
\hline 1 & $\begin{array}{c}9011761052412 \\
8141331\end{array}$ & 134 & 586 & 808 & 95 & 347.5 & 134 & 589 & 802 & 95 & 346.9 & 0.6 & 58 & 29.3 \\
\hline 2 & $\begin{array}{c}6511408721043 \\
1312911\end{array}$ & 127 & 523 & 738 & 37 & 301.4 & 127 & 529 & 732 & 37 & 301.4 & 0 & 60 & 30 \\
\hline 3 & $\begin{array}{c}1230101625814 \\
7913411\end{array}$ & 126 & 556 & 728 & 38 & 306 & 126 & 571 & 722 & 38 & 307.8 & 1.8 & 86 & 43.9 \\
\hline 4 & $\begin{array}{c}01761113122105 \\
398414\end{array}$ & 129 & 594 & 752 & 91 & 335.2 & 129 & 594 & 752 & 91 & 335.2 & 0 & 73 & 36.5 \\
\hline 5 & $\begin{array}{c}4690251278310 \\
1311411\end{array}$ & 123 & 611 & 698 & 49 & 313.4 & 123 & 611 & 692 & 49 & 312.2 & 1.2 & 71 & 36.1 \\
\hline \multirow[t]{2}{*}{6} & $\begin{array}{c}10111292045143 \\
813716\end{array}$ & 136 & 554 & 828 & 87 & 343.3 & 136 & 552 & 822 & 86 & 341.4 & 1.9 & 95 & 48.45 \\
\hline & average & & & & & 324.46 & & & & & 324.15 & 0.91 & 73.83 & 37.37 \\
\hline
\end{tabular}

Let us compare all the simulations carried out by the HMOIA II. The best schedule for dealing with the disruption (the best value of FFry*) was achieved in the first simulation for affthres $=80$. The best basic schedule was obtained by the HMOIA II (Figure 5).

Better basic schedules were also achieved for affthres $=80$ taking into account the average values of FFry*. The same conclusion can be given taking into account the average values of FFy for basic schedules. 


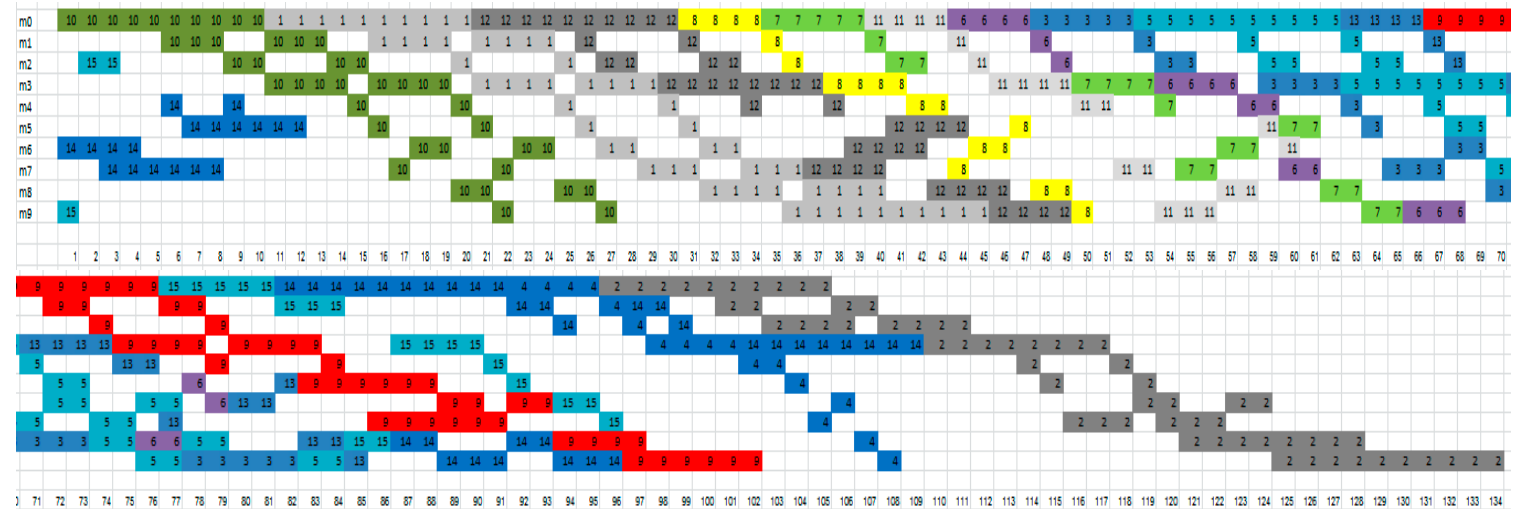

Figure 5. The best basic schedule obtained by the MOIA II (affthres $=8$ ) and MIDOS, where $(i=\{0,2, \ldots, 9\}$ is no. of machine, $(j=\{1,2, \ldots, 15\}$ is no. of job, 99 is predicted technical inspection at time 66 .

\subsection{Discussion}

The following research point was investigated in this paper-which construction of proactive algorithms achieves better solutions: proactive-reactive (proactive without prediction) or predictive-reactive (proactive with prediction). The first construction consists of repeating the closed loop: the task sequences population is randomly selected, trained and evaluated measuring the effects of the disturbance on quality robustness QR and solution robustness SR. Proactive-reactive (proactive without prediction) simulations were run using the HMOIA II. The second construction consists of repeating the closed loop: a population of the task sequence is generated, and the sequence is rebuilt using the MIDOS rule in order to make the schedule more robust. Next, the schedules were trained and evaluated separately taking into account the effects of the disturbance on QR and SR. Predictive-reactive (proactive with prediction) simulations were run using the HMOIA III.

After the comparison analysis of proactive methods, the following conclusion can be given. The proactive-reactive approach achieves less robust schedules than the predictive-reactive approach. The predictive-reactive approach achieves better schedules for the weighted sum of SR and QR. The average value of the weighted sum of SR and QR obtained in the predictive-reactive approach FFrx ${ }^{*}$ equals 13.71 (for affthres $=8$ ) and 12.49 (for affthres $=80$ ). The average value of the weighted sum of SR and QR obtained in the proactive-reactive approach FFry* equals 45.03 (for affthres $=8$ ) and 37.37 (for affthres $=80$ ).

The presented approaches were compared in order to select a better method of production organization that reduces costs and waste due to machine failure. Both criteria QR and SR are used in order to compute the operational efficiency of the production system in the event of disruption. Any cost criterion can be added to the QR in order to measure losses due to a machine failure. The proactive-reactive approach achieves better schedules for the QR criterion. The average value of QR equals 0.91 (for affthres $=80$ ) (Table 4 ).

The SR criterion measures a number of changes necessary to adopt the production schedule to a new situation (after the machine failure). Additional energy is consumed by adapting of a shop floor in accordance with the schedule changes. Additional set-up operations are carried out for machines. Additional organizational changes of human resources and raw material requirements are also necessary. The predictive-reactive approach achieves better schedules for SR criterion. The average value of SR equals 22.08 (for affthres $=80$ ) (Table 2 ).

The predictive-reactive approach is better in the process of seeking a compromise solution for the two criteria SR and QR.

The proactive-reactive approach presented in this paper can be compared with the approach based on the tabu search algorithm (TSA) [25]. "TSA accepts the value of parameters generating the 
most robust schedules. The scheduling algorithm consists of two parts, i.e. a sequence generator and a sequence evaluator. TSA is applied to effective scanning of the solution space." [25]. The average slack method (ASM) "is applied to evaluate a reactive schedule using the same criterion as the criteria used for the basic schedule evaluation. However, in this case this criterion is increased by the value of deterioration of the criterion due to a disturbance." [25]. The best basic schedule was generated according to the rule of $\{25814104671011123913\}$. The quality of the reactive schedule is $\mathrm{FFr}_{2^{*}}=$ 90.5 with the components of $\mathrm{QR}=3$ and $\mathrm{SR}=178$. The quality of this schedule is also measured using $C_{\max }, F, I$ and $T$. The quality of the basic schedule is $C_{\max }=117, F=516, I=638$ and $T=0$, and $C_{\max }=$ $117, F=507, I=632$ and $T=0$ after rescheduling. The average values of FFry* for obtained schedules equals 132.25 .

The following conclusion can be given taking into account the average values of FFry* and the best schedule achieved using the TSA. The proactive-reactive approach presented in this paper is better than the approach based on the TSA. The proactive-reactive approach presented in this paper achieves more stable and robust schedules. However, the proactive-reactive approach presented in [25] achieves schedules with non-delayed jobs even after disruption.

The predictive-reactive approach presented in this paper can be compared with the approach based on the multi-objective immune algorithm (MOIA) and the clonal selection algorithm (CSA) [6,24]. In the MOIA, two steps are distinguished: the exogenous and endogenous activations that imitate the immune system. The CSA imitates the affinity maturation process. The MOIA and CSA are applied to the same JS system. Basic solutions are evaluated using criteria $C_{\max }, F, I$ and $T$. To get the best predictive schedules, the MIDOS rule for the best basic solutions is used. Reactive schedules are generated by the use of rescheduling policies. After disruption, the performance of the job shop system is investigated using the rule of MIROS. The influence of the disruption is evaluated using SR and QR. The predictive schedule generated by the MIDOS and the priority rule obtained by the CSA absorbs the influence of the disruption more efficiently. The best basic solution was obtained according to the priority rule of $\{10142531047896111213\}$. The quality of the reactive solution is $\mathrm{FFr}_{3^{*}}=5.5$ with the sub-functions of $\mathrm{QR}=4$ and $\mathrm{SR}=7$. The quality of the basic solution is: $C_{\max }=116, F=613, I=622$ and $T=11$, and $C_{\max }=116, F=608, I=619$ and $T=11$ after disruption. The average value of FFrx* equals 12.25 for the two best schedules obtained using the MOIA. The average value of FFrx* equals 2.75 for the two best schedules obtained using CSA.

The following conclusion can be given taking into account the average values of FFrx* achieved using the MOIA and CSA. The predictive-reactive approach presented in this paper achieves similar solutions as the approach based on the MOIA [24]. The predictive-reactive approach presented in this paper achieves worse solutions compared to the approach based on the CSA [24]. The following conclusion can be given taking into account the best schedule achieved using the MOIA and CSA. The predictive-reactive approach presented in this paper achieved the most stable and robust schedule.

\section{Conclusions}

This paper presents a comparison of methods of dealing with uncertainty in scheduling problems. The paper is a response to the need for "sustainable manufacturing". Researchers are searching for opportunities to organize production systems that save energy and natural resources. Preventive maintenance (PM) is essential for the efficient use of machines and energy saving. Any rework due to a machine failure consumes additional energy, human resources, equipment, spare parts and raw materials. Criteria $\mathrm{QR}$ and SR are used in order to compute the operational efficiency of the production system in the event of disruption. The presented approaches were compared in order to select a better method of production organization that reduces costs and waste due to machine failure.

Two proactive approaches were compared: predictive-reactive (proactive with prediction) and proactive-reactive (proactive without prediction). In the predictive-reactive approach, the time of PM is predicted and a predictive schedule is built. Next, the influence of disturbance on the predictive schedule using robustness measures is examined. In the proactive-reactive approach the proactive 
schedule is achieved for the best sequence of idle times between jobs taking advantage of the simulation process. Next, the influence of disturbance on the proactive schedule using robustness measures is examined. This paper presents the results of computer simulations for the above approaches. The main conclusions based on the study presented are:

- comparing two approaches, predictive-reactive and proactive-reactive, the first method provides more compromise schedules for mean weighted values of QR and SR functions;

- the presented predictive-reactive approach achieved the best quality schedule for the stability and robustness criteria, SR and QR.

The predictive schedules obtained in the results of computer simulations indicate that the scheduling method based on meta-heuristic (immune algorithm) is suitable for application in actual production processes. The results of the study were achieved in short time. In addition, the criteria used to evaluate schedules, $C_{\max }, F, I$ and $T$, are the most popular not only in academic practice. Gantt charts present the assignment of tasks to machines in a transparent manner and are easy to analyze taking into account the evaluation criteria.

Practitioners want a schedule to be more robust for actual events regarding production systems. Therefore, the anticipated failure-free time of a machine is included in the schedule. The failure-free time is estimated by using the method based on probability theory. Incorporation of the historical failure-free times of machines into the prognostic process makes the prognosis more accurate. The risk of inconsistent prediction of machine failure time is minimized by assigning the most flexible operations to the period of higher probability of failure. The MIDOS rule assigns operations that introduce the smallest number of changes into the schedule during a high probability of machine failure.

The forecast of failure-free time can be updated after every equal period of time, for example, every 8,16 or $24 \mathrm{~h}$. The update prevents errors resulting from inconsistency between the anticipated failure-free time and the actual failure time. The schedule can be updated after each failure or after an equal time.

The author of this paper intends to develop the existing research by comparing methods of predicting the failure-free time of a machine, that is, the maximum likelihood, empirical moments and a method based on renewal theory. The purpose of the comparison is to determine for which method it is possible to obtain more accurate predictions. Accurate predictions are the key to reliable scheduling.

Funding: This research was funded from the statutory grant of the Faculty of Mechanical Engineering of the Silesian University of Technology: 10/990/BK18/0099.

Conflicts of Interest: The author declares no conflict of interest.

\section{References}

1. Wang, S.; Yu, J. An effective heuristic for flexible job-shop scheduling problem with maintenance activities. Comput. Ind. Eng. 2010, 59, 436-447. [CrossRef]

2. Rajkumar, M.; Asokan, P.; Vamsikrishna, V. A GRASP algorithm for flexible job-shop scheduling with maintenance constraints. Int. J. Prod. Res. 2010, 48, 6821-6836. [CrossRef]

3. Liu, L.; Gu, H.Y.; Xi, Y.G. Robust and stable scheduling of a single machine with random machine breakdowns. Int. J. Adv. Manuf. Technol. 2007, 31, 645-656. [CrossRef]

4. Herroelen, W.; Leus, R. Project scheduling under uncertainity: Survey and research potentials. Eur. J. Oper. Res. 2005, 165, 289-306. [CrossRef]

5. Hasan, S.M.K.; Sarker, R.; Essam, D. Genetic algorithm for job-shop scheduling with machine unavailability and breakdowns. Int. J. Prod. Res. 2011, 49, 4999-5015. [CrossRef]

6. Paprocka, I.; Kempa, W.M. Searching for a Method of Basic Schedules Generation which influences over the Performance of Predictive and Reactive Schedules. In Information Systems Architecture and Technology, Proceedings of the 37th International Conference on Information Systems Architecture and Technology-ISAT 2016-Part IV, Karpacz, Poland, 18-20 September 2016; Advances in Intelligent Systems and Computing; Springer: New York, NY, USA, 2016; Volume 524, pp. 233-242. 
7. Paprocka, I. The model of maintenance planning and production scheduling for maximizing robustness. Int. Prod. Res. 2018. [CrossRef]

8. Cui, W.W.; Lu, Z.; Pan, E. Integrated production scheduling and maintenance policy for robustness in a single machine. Comput. Oper. Res. 2014, 47, 81-91. [CrossRef]

9. Cui, W.W.; Lu, Z.; Li, C.; Han, X. A proactive approach to solve integrated production scheduling and maintenance planning problem in flow shops. Comput. Ind. Eng. 2018, 115, 342-353. [CrossRef]

10. Xia, T.; Jin, X.; Xi, L.; Ni, J. Production-driven opportunistic maintenance for batch production based on MAM-APB scheduling. Eur. J. Oper. Res. 2015, 240, 781-790. [CrossRef]

11. Zhang, L.; Gao, L.; Li, X. A hybrid genetic algorithm and tabu search for multi-objective dynamic job shop scheduling problem. Int. J. Prod. Res. 2013, 51, 3516-3531. [CrossRef]

12. Lei, D. Scheduling fuzzy job shop with preventive maintenance through swarm-based neighborhood search. Int. J. Adv. Manuf. Technol. 2011, 54, 1121-1128. [CrossRef]

13. Bali, N.; Labdelaoui, H. Optimal Generator Maintenance Scheduling Using a Hybrid Metaheuristic Approach. Int. J. Comput. Intell. Appl. 2015, 14, 1550011. [CrossRef]

14. Mokhtari, H.; Mozdgir, A.; Abadi, I.N.K. A reliability/availability approach to joint production and maintenance scheduling with multiple preventive maintenance services. Int. J. Prod. Res. 2012, 50, 1-20. [CrossRef]

15. Bajestani, M.A.; Banjevic, D.; Beck, J.C. Integrated maintenance planning and production scheduling with Markovian deteriorating machine conditions. Int. J. Prod. Res. 2014, 52, 7377-7400. [CrossRef]

16. Sobaszek, Ł.; Gola, A.; Świć, A. Predictive scheduling as a part of intelligent job scheduling system. Adv. Intell. Syst. Comput. 2018, 637, 358-367.

17. Kempa, W.M.; Paprocka, I.; Kalinowski, K.; Grabowik, C. Estimation of reliability characteristics in a production scheduling model with failures and time-changing parameters described by Gamma and exponential distributions. Adv. Mater. Res. 2014, 837, 116-121. [CrossRef]

18. Skołud, B.; Wosik, I.; Wosik, I. Multi-objective genetic and immune algorithms for batch scheduling problem with dependent setups. Recent Dev. Artif. Intell. Methods 2007, 185-196.

19. Skołud, B.; Wosik, I. The development of IA with local search approach for multi-objective Job shop scheduling problem. In Virtual Design and Automation; Publishing House of Poznań University of Technology: Poznań, Poland, 2008; pp. 235-242.

20. Skołud, B.; Wosik, I. Clonally selection and multi-objective immune algorithms for open job shop scheduling problems. In Proceedings of the 30th International Conference Information Systems, Architecture, and Technology, System Analysis in Decision Aided Problems, Wrocław, Poland, 20-22 September 2009; pp. 217-230.

21. Paprocka, I.; Gwiazda, A.; Baczkowicz, M. Application of the Hybrid-Multi Objective Immune Algorithm for obtaining the robustness of schedules. In Proceedings of the International Joint Conference SOCO'16-CISIS'16-ICEUTE'16, San Sebastián, Spain, 19-21 October 2016; Volume 527, pp. 445-453.

22. Paprocka, I.; Skołud, B. A Hybrid-Multi Objective Immune Algorithm for predictive and reactive scheduling. J. Sched. 2017, 20, 165-182. [CrossRef]

23. Kempa, W.M.; Wosik, I.; Skołud, B. Estimation of reliability characteristics in a production scheduling model with time-changing parameters-First part, theory. Manag. Control Manuf. Process. 2011, 7-18.

24. Paprocka, I. On the Quality of Basic Schedules Influencing over the Performance of Predictive and Reactive Schedules. In Information Systems Architecture and Technology, Proceedings of 37th International Conference on Information Systems Architecture and Technology-ISAT 2016-Part IV, Karpacz, Poland, 18-20 September 2016; Advances in Intelligent Systems and Computing; Springer: New York, NY, USA, 2016; Volume 524, pp. 243-253.

25. Paprocka, I.; Gwiazda, A.; Baczkowicz, M. Robustness of schedules obtained using the Tabu search algorithm based on the Average Slack Method. In Proceedings of the International Joint Conference SOCO'16-CISIS'16-ICEUTE'16, San Sebastián, Spain, 19-21 October 2016; Volume 527, pp. 426-434.

(C) 2018 by the author. Licensee MDPI, Basel, Switzerland. This article is an open access article distributed under the terms and conditions of the Creative Commons Attribution (CC BY) license (http:/ / creativecommons.org/licenses/by/4.0/). 